\title{
TENDENCIA ANUAL DEL CAUDAL DE SALIDA, EN REFERENCIA AL CAUDAL ECOLÓGICO EN LA MICROCUENCA APACHETA / AYACUCHO / PERÚ, DEL 2000 AL 2018
}

\author{
OUTFLOW ANNUAL TREND, COMPARED TO THE ECOLOGICAL \\ FLOW, IN THE APACHETA MICRO-BASIN / AYACUCHO / PERU, \\ FROM 2000 TO 2018
}

\author{
Wilmer Moncada ${ }^{1}$ y Bram Willems ${ }^{2}$
}

\begin{abstract}
Resumen
El decreciente caudal de salida en la microcuenca Apacheta, observado durante el periodo 20002018, afecta de manera directa el almacenamiento de agua en la presa Cuchoquesera e impacta, de manera negativa, en la demanda para consumo humano y agrícola, muchas veces por encima de su disponibilidad hídrica, así como en el buen estado ecológico de la microcuenca. A largo plazo, este problema puede intensificarse a consecuencia del cambio climático. El objetivo del presente estudio fue evaluar la tendencia anual del caudal de salida en la microcuenca Apacheta, así como pronosticar valores futuros, en referencia a su caudal ecológico de $2.24 \mathrm{~m}^{3} / \mathrm{s}$ y su sensibilidad en el momento de satisfacer adecuadamente las diferentes demandas. Para ello, se combinaron datos hidrometeorológicos, información satelital y el modelo PRMS-IV. Nuestros resultados indican que, de continuar la tendencia anual del caudal de salida, se podría esperar una disminución del $46.1 \%$ y $56.1 \%$ por debajo de su caudal ecológico, para los próximos 5 y 10 años, respectivamente. A nivel de gobiernos regionales y nacionales, estos plazos se consideran típicamente para fines de planificación, en ese sentido, nuestros resultados tienen el potencial de guiar procesos de toma de decisiones hacia la mitigación de riesgos de escasez de agua.
\end{abstract}

Palabras clave: tendencia, escasez hídrica, Apacheta, PRMS, caudal, ecológico.

\begin{abstract}
Decreasing outflow observed in the Apacheta micro-basin during the period 2000-2018, has a direct impact on the water storage of the Cuchoquesera dam, with subsequent negative impacts on local water provisioning for human consumption and agricultural activities - whose demand has already surpassed the supply capacity of the reservoir - as well as the ecological volume needed for ensuring the proper functioning of ecosystems along the catchment. In the long term, this problem may be intensified by the effects of climate change. The objective of this study was to assess the annual trend of the Apacheta micro-basin discharge and forecast future values, in comparison to its ecological flow of $2.24 \mathrm{~m}^{3} / \mathrm{s}$ and the sensitivity towards adequately satisfying the different demands over time. For this, we combined hydro-meteorological data and remote sensing information with the PRMS-IV hydrological model. Our results indicate that, upon continuing the annual outflow trend, a decrease of $46.1 \%$ and $56.1 \%$ below the ecological flow could be expected for the next 5 and 10 years, respectively. At the regional and national government levels, timeframes of 5-10 years are typically considered for planning purposes, in that sense, our results have the potential to guide decision-making processes towards mitigating water-scarcity risks.
\end{abstract}

Key words: trend, water-scarcity, Apacheta, PRMS, flow, ecological.

\section{Introducción}

Es posible que en la actualidad la microcuenca Apacheta de la Región Ayacucho, Perú, se encuentre consumiendo sus reservas hídricas, en el sentido de que la permanencia de nieve en los nevados ha ido disminuyendo en los últimos años, probablemente debido a la influencia del cambio climático (Pereda et al., 2018). En los últimos años, la sostenibilidad de los ecosistemas de humedales en la microcuenca se ha visto afectada por el incremento de la temperatura y la disminución de la precipitación que impactan en los bofedales $\mathrm{y}$, por ende, en la reducción de las concentraciones de carbono (Huaman et al., 2020). Asimismo, el deshielo de los nevados, que se traduce en escorrentía y escurrimiento superficial y subsuperficial a través de tributarios al rio Apacheta, contribuyen vía trasvase a la presa Cuchoquesera para el consumo poblacional de la ciudad de Huamanga e irrigación de áreas agrícolas en la cuenca Cachi (Moncada et al., 2018). 
La aplicación del Sistema de Modelado de Precipitación y Escorrentía, versión 4 (PRMS-IV), a la cuenca del río Apalachicola-Chattahoochee-Flint en el sureste de Estados Unidos (1950 a 1999) simuló el caudal de salida mediante forzamientos climáticos, evaluado y calibrado con parámetros derivados de datos espaciales y datos medidos en 35 medidores de flujo USGS. Los resultados validados con el Índice de Eficiencia Nash-Sutcliffe muestran un representativo balance de masa de la cuenca (LaFontaine et al., 2013). Estos resultados demuestran que la aplicación del modelo PRMS-IV en cualquier cuenca puede lograr un balance de masa confiable en el proceso de precipitación y escorrentía (Teng et al., 2017).

En efecto, para la determinación del balance de masa y la evaluación del caudal de salida en la microcuenca Apacheta, el modelo PRMS-IV requiere de datos climáticos e información geográfica, además de validar los resultados con datos de aforo medidos en la estación hidrometeorológica Apacheta, administrada por la Oficina de Operaciones y Mantenimiento de la cuenca Cachi del Gobierno Regional de Ayacucho (OPEMAN). La administración de los datos es útil para el seguimiento y sustento de una propuesta de protección y mantenimiento de la microcuenca Apacheta, enmarcadas dentro del ya culminado proyecto integral del rio Cachi (Corporación de Fomento y Desarrollo Económico y Social de Ayacucho, 1983).

Para lograrlo, se requiere del manejo de datos climáticos y la obtención de información a partir del uso de técnicas de teledetección satelital (Sobrino, 2000; Canty, 2014) en la determinación de la cobertura de vegetación (Rouse et al., 1974), la cobertura nival (Dozier, 1989), elevación del terreno, relieve, orientación, aspecto y mapa de sombras extraídas de un Modelo de Elevación Digital (DEM) (Cook et al., 2012).

De acuerdo con Kult et al. (2014) y Markstrom et al. (2015), el balance de masa obtenido del modelo PRMS-IV se calibra manipulando y ajustando parámetros de calibración y se valida con datos de aforo. El balance de masa estimado permite evaluar la estacionalidad, el comportamiento de la tendencia anual y la variación del caudal de salida para la determinación de escenarios futuros en referencia al caudal ecológico de la microcuenca Apacheta.

A partir de la serie de tiempo del caudal medio mensual de salida en la microcuenca Apacheta, se determina el caudal ecológico o caudal mínimo equivalente al caudal, de acuerdo con la metodología que aprueba los "Lineamientos generales para determinar caudales ecológicos" detallada en la Resolución Jefatural N 267-2019-ANA (ANA, 2019).

En este sentido, el objetivo del presente trabajo fue evaluar la tendencia anual y pronóstico del caudal de salida en referencia al caudal ecológico de la microcuenca Apacheta, Región Ayacucho, desde el año 2000 al 2018.

\section{Materiales y Métodos}

La microcuenca Apacheta es cabecera de la cuenca Cachi, en el distrito de Paras, provincia de Cangallo, Región Ayacucho, Perú, con longitudes de $74^{\circ} 37^{\prime}$ $26.4^{\prime \prime} \mathrm{W}$ a $74^{\circ} 45^{\prime} 32.4^{\prime \prime} \mathrm{W}$ y latitudes de $13^{\circ} 17^{\prime} 6^{\prime \prime} \mathrm{S}$ a $13^{\circ} 26^{\prime} 9.6^{\prime \prime} \mathrm{S}$. La línea limítrofe mostrada en la Figura 1 separa el área sur en verde claro perteneciente a la Región Ayacucho del área norte en verde oscuro perteneciente a la provincia de Huaytará de la Región Huancavelica. Tiene un área de 14348.21 ha, su elevación mínima es de 4112 msnm, su elevación máxima de $5045 \mathrm{msnm}$, y su elevación media es de 4 $651 \mathrm{msnm}$ (Pereda et al., 2018). Se caracteriza por tener tres estadíos climáticos, el lluvioso con valores altos de precipitación, temperatura mínima del aire y humedad; el estadío seco, con valores altos de velocidad del viento y bajos de temperatura mínima del aire; el estadío intermedio, con valores altos de temperatura máxima del aire y radiación solar, con valores medios de precipitación $71.09 \mathrm{~mm}$, temperatura máxima del aire $12.73{ }^{\circ} \mathrm{C}$, temperatura mínima del aire $-0.35{ }^{\circ} \mathrm{C}$, velocidad del viento $2.91 \mathrm{~m} / \mathrm{s}$ y radiación solar 514.43 Ly (Moncada et al., 2020).

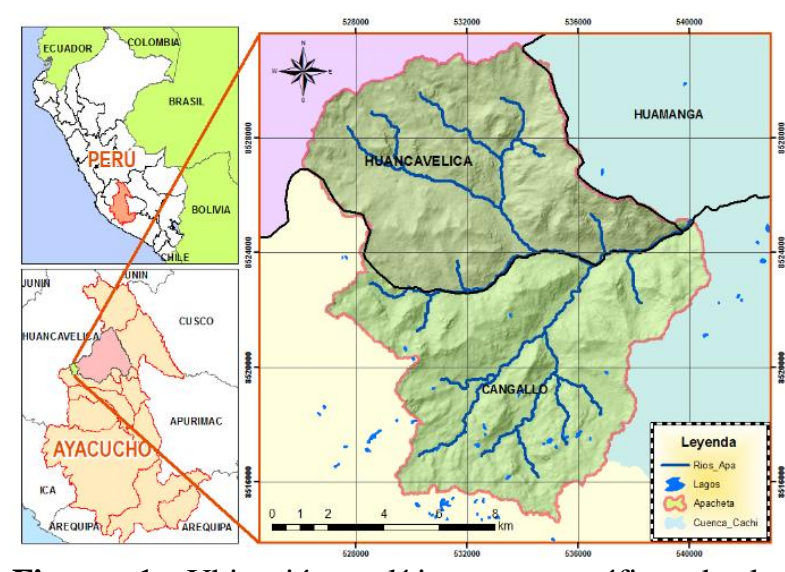

Figura 1. Ubicación política y geográfica de la microcuenca Apacheta, Región Ayacucho, Perú (Pereda et al., 2018).

El modelo PRMS-IV es un sistema de modelado basado en procesos físicos, determinista y de parámetros distribuidos, desarrollado para evaluar la respuesta de varias combinaciones de clima y uso de suelo en la hidrología de cuencas hidrográficas, originalmente descrito por Leavesley et al. (1983); simula procesos del ciclo hidrológico, que incluyen el caudal de salida, la evapotranspiración potencial, escorrentía, infiltración e interflujo estimados del balance de masa y energía a partir de datos de cobertura de vegetación, cobertura nival, zona de suelo e información climática, distribuida en 31 unidades de respuesta hidrológica (HRU). Los datos de entrada al 
modelo PRMS-IV son valores diarios de precipitación, velocidad del viento, temperatura máxima y mínima del aire y datos de caudal de la estación hidrometeorológica Apacheta. También se requieren datos diarios de radiación solar de onda corta extraídos del sistema de asimilación de datos terrestres globales versión 2 (GLDAS-2) de la plataforma Giovanni NASA. El esquema de la Figura 2 muestra la metodología a seguir para determinar el balance de masa con el modelo PRMS-IV.

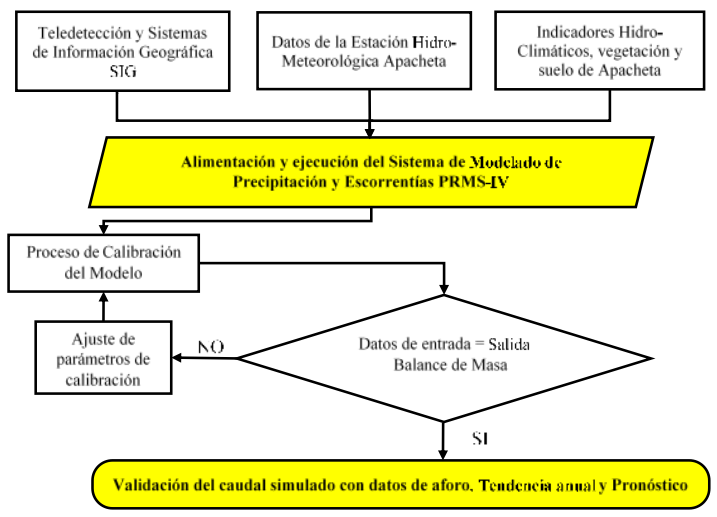

Figura 2. Esquema metodológico de la aplicación del modelo PRMS-IV para la simulación del caudal de salida en la microcuenca Apacheta, Región Ayacucho, Perú.

El modelo PRMS-IV se ejecutó mediante módulos especificados por el usuario en la secuencia correcta para simular el ciclo hidrológico completo; incluye el cálculo de la distribución climática, período de transpiración activa, evapotranspiración potencial, intercepción de la precipitación en la cubierta foliar, acumulación de capas de nieve y derretimiento, escorrentía superficial e infiltración, cuantificación de la humedad en el suelo, flujo de agua subterránea, enrutamiento del caudal y almacenamiento de la superficie en depresiones. Estos módulos, junto con otros, se utilizaron para la configuración del modelo, entrada de datos e informes de salida, con ecuaciones pertinentes y tablas que describen las entradas y salidas mediante parámetros (Markstrom et al., 2008).

En la calibración del modelo se utilizó la técnica de Evolución de Mezcla Compleja (Duan et al., 1994). Se realizó de manera manual siguiendo el procedimiento de calibración paso a paso. Primero se calibró la radiación solar, por ejemplo, con el parámetro "dday_intcp" (con valores entre -70 y 10); después se calibra la evapotranspiración potencial con el parámetro "jh_coef" (con valores entre 0.005 y 0.09 ); seguidamente se calibró el volumen de caudal, por ejemplo, con el parámetro "adjust_rain" (con valores entre 0 y 1 ); por último, se calibró el tiempo de caudal, por ejemplo, con el parámetro "adjmix rain" (con valores entre 0 y 1). Este procedimiento se repitió hasta que se encontró el ajuste óptimo entre los valores simulados y observados, mediante una función objetivo o de optimización de acuerdo con el coeficiente de ajuste en cada paso (Chang \& Jung, 2010). Para ello, se utilizó el caudal diario del limnímetro de la bocatoma Apacheta, desde el 01 de setiembre de 2004 al 30 de mayo de 2017.

El caudal de salida simulado se validó con datos de aforo medidos en la coordenada UTM, latitud 539857 $\mathrm{m}$ y longitud $8524841 \mathrm{~m}$, en la bocatoma Apacheta, desde el 27 de marzo de 2009 al 17 de mayo de 2017, con un promedio de 9 a 10 datos por año, haciendo un total de 85 datos. El grado de confiabilidad del caudal simulado se determinó calculando la eficiencia de Nash-Sutcliffe, el coeficiente de Nash-Sutcliffe-Ln para los valores del logaritmo (Krause et al., 2005), el coeficiente de correlación de Pearson, el coeficiente de determinación $\mathrm{R}^{2}$, la raíz del error medio cuadrático (RMSE), Bias Score (BS), PBias, el error relativo al volumen (RVE) y el error de valores picos normalizado (NPE) (Nash \& Sutcliffe, 1970; Krause et al., 2005).

Para la determinación del caudal medio, se calculó el volumen debajo de la curva de duración del caudal con el código Matlab (Apéndice 1), a partir de la serie de tiempo del caudal simulado con PRMS-IV en la microcuenca Apacheta.

Se aplicó la prueba de normalidad de KolmogorovSmirnov con la corrección Lilliefors a la serie de tiempo del caudal medio mensual de salida simulado, donde: $\mathrm{H}_{0}$ indica que los datos del caudal medio mensual provienen de una distribución normal; $\mathrm{H}_{1}$ indica que los datos del caudal medio mensual no provienen de una distribución normal (Lilliefors, 1967). Utilizando el software $R$ se ejecutó la prueba Lilliefors con el paquete "nortest", ejecutando el código "lillie.test", y se obtuvo el valor de discrepancia D = 0.148 , que correspondió a la discrepancia máxima en valor absoluto entre los datos medidos y teóricos, con un $\mathrm{p}$-valor $=2.997 \times 10^{-13}$, menor al nivel de significancia $\alpha=0.05$, por lo que se rechazó la hipótesis nula $\mathrm{H}_{0}$, lo que significa que los datos del caudal medio mensual no provienen de una distribución normal. Este resultado permitió tener en cuenta el sesgo del pronóstico.

Con el código del Apéndice 2, en R, se realizó el pronóstico del caudal de salida en la microcuenca Apacheta (Tornero, 2017). La función "forecast" emplea "auto.arima", que ajusta los datos del caudal medio mensual simulado de enero de 2000 a diciembre de 2018, al mejor modelo $\operatorname{ARIMA}(\mathrm{p}, \mathrm{d}, \mathrm{q})$; donde p, es el término de la componente auto regresiva (AR); d, es el término de la componente diferencial (I); y q, es el término de la componente de media móvil (MA), para series de tiempo estacionales (Hyndman et al., 2020). El paquete "prophet" utiliza modelos de regresión aditivo, que ajusta los parámetros de estacionalidad, cambios de tendencia y capacidad de crecimiento o irregularidades atípicas, generando un posible 
escenario futuro de 24 meses, automatizado y robusto (Taylor \& Letham, 2017).

En la Tabla 1 se muestra la plantilla de cálculo del caudal ecológico para los $\mathrm{N}=19$ años de datos de caudal medio mensual simulados, del año 2000 al 2018. En la segunda columna se observan las probabilidades calculadas con: $\mathrm{P}(\%)=(\mathrm{m} / \mathrm{N}) \times 100$, ordenadas de menor a mayor, correspondientes a los caudales medios para cada mes de mayor a menor (ANA, 2019).

Los valores del caudal ecológico corresponden al $95 \%$, que se calcularon por interpolación lineal con los valores adyacentes, al $94.74 \%$ y $100 \%$, los cuales se muestran en la Tabla 2. Estos valores se interpolaron al 95\% de persistencia en el punto de interés de la fuente natural de agua, dando un valor medio de $2.24 \mathrm{~m}^{3} / \mathrm{s}$ de caudal ecológico en la microcuenca Apacheta.

\section{Resultados y discusión}

Las pruebas de validación de la Tabla 3, realizadas al caudal simulado con PRMS-IV respecto al caudal observado o medido en el punto de aforo en la bocatoma Apacheta desde el 27 de marzo de 2009 al 17 de mayo de 2017, un promedio de 9 a 10 datos por año, con un total de 85 datos, muestra que los valores reales calculados por los indicadores se encuentran dentro del rango de evaluación y muy cerca del valor ideal.

En complemento a las pruebas de validación de la Tabla 3, se compararon los datos del caudal de salida simulado con el caudal observado o medido en el punto de aforo en la bocatoma Apacheta, tal como se muestra en la Figura 3. Se observa que ambos guardan correspondencia en su comportamiento y un buen ajuste para fechas diferidas del año 2009 al 2017.

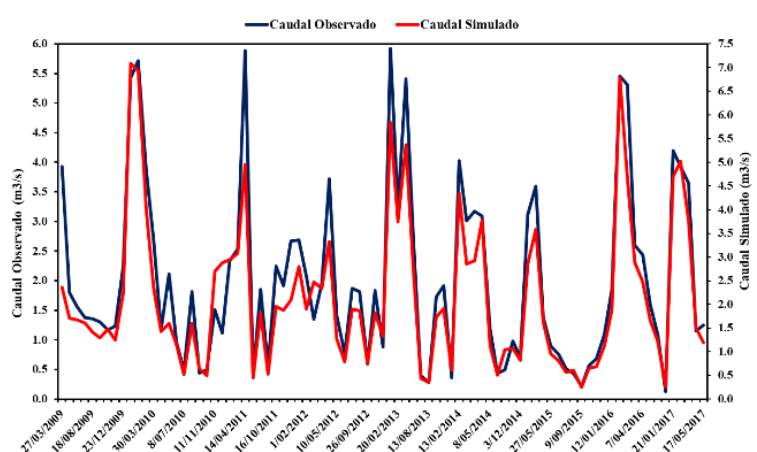

Figura 3. Comparación del caudal simulado con el caudal observado de salida en la microcuenca Apacheta, Región Ayacucho.

La validación del caudal de salida simulada se da en relación con los datos de aforo del caudal observado de manera gráfica, conforme se muestra en la Figura 4. La correlación lineal se establece mediante un coeficiente de determinación $\mathrm{R}^{2}=0.91$, lo cual significa que los datos del caudal observado explican en un $91 \%$ al caudal de salida simulado con PRMS-IV. Esta aseveración, en adición a las pruebas de validación de la Tabla 3, es suficiente para afirmar que el modelo funciona correctamente y que el resultado es válido con una ligera sobre estimación del caudal simulado con respecto al observado, en relación con la línea de tendencia.

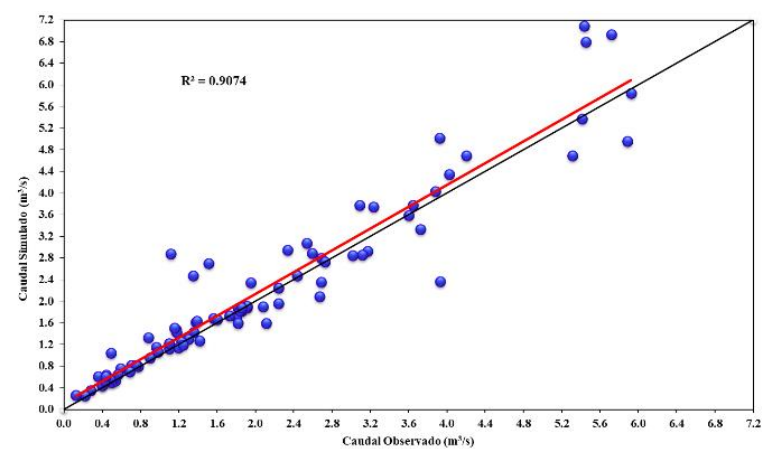

Figura 4. Correlación lineal entre el caudal simulado con el modelo PRMS IV con respecto al caudal observado medidos en la bocatoma Apacheta.

La simulación del caudal diario de salida en la microcuenca, desde el 01 de enero del 2000 hasta el 31 de diciembre del 2018, se muestra en la Figura 5. Esta serie de tiempo permite construir la curva de duración de flujo de caudal diario de salida en la bocatoma Apacheta. La prueba de normalidad aplicada a la serie de tiempo del caudal simulado indica que los datos no provienen de una distribución normal ( $\mathrm{p}<0.05)$; por lo tanto, el pronóstico del comportamiento del caudal en el tiempo deseado se obtiene con un sesgo de pronóstico muy alto, por lo que la curva de duración es típica para el régimen de caudales.

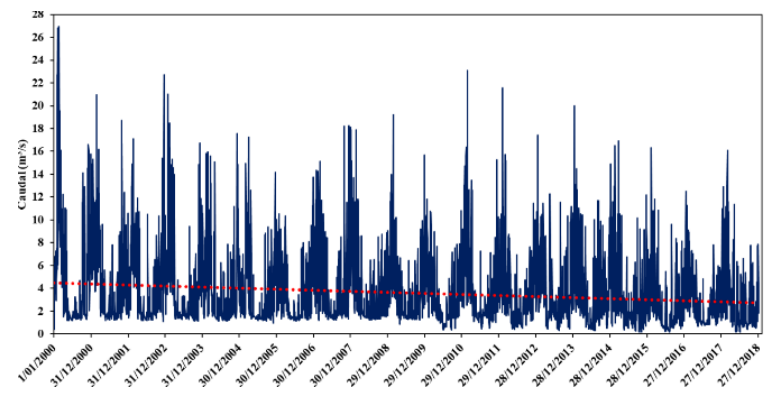

Figura 5. Serie de tiempo del caudal diario de salida simulado con el modelo PRMS-IV en la microcuenca Apacheta, del año 2000 al 2018.

En la Figura 6 se observa la escala vertical de la curva de duración del caudal diario $\left(\mathrm{m}^{3} / \mathrm{s}\right)$ y la escala horizontal para la probabilidad de que el caudal pueda ser igualado o excedido, es decir el porcentaje (\%) de tiempo que indica que el caudal fue igualado o superado. Se aprecia que la curva de duración de caudal tiene una forma típica que depende de las particularidades de la microcuenca, donde la pendiente acentuada en el tramo inicial de la curva de duración indica que se presenta, con menor probabilidad, un caudal máximo de $27 \mathrm{~m}^{3} / \mathrm{s}$ en períodos muy cortos. El 
registro de datos diarios utilizados para la construcción de la curva de duración de flujo de caudal corresponde a la serie de tiempo de la Figura 5; por lo tanto, cuanto mayor es el número de registros mayor será la confiabilidad de la estimación, siempre que las condiciones de la microcuenca se mantengan sin cambios considerables. La forma de la curva de duración del caudal de salida es un indicativo del proceso de drenaje, es decir, presenta una pendiente pronunciada entre el $0 \%$ y el $50 \%$ de excedencia, la misma que representa una corriente de agua con gran variabilidad entre los caudales transportados; esto significa que su caudal proviene principalmente de la escorrentía superficial. El valor del caudal que se obtiene para el $50 \%$ del tiempo igualado o excedido corresponde a la mediana del caudal de la serie, equivalente a $2.27 \mathrm{~m}^{3} / \mathrm{s}$. El valor calculado de $\mathrm{Z}=1$ 316.8 corresponde al valor de la integral debajo de la curva de duración de caudal que se divide entre 365 días, da un caudal medio de $3.61 \mathrm{~m}^{3} / \mathrm{s}$, que es mayor a la mediana del caudal de $2.27 \mathrm{~m}^{3} / \mathrm{s}$, lo que significa que existen caudales mayores a $3.61 \mathrm{~m}^{3} / \mathrm{s}$ en porcentajes de tiempo menores a $34.37 \%$. Asimismo, existe mayor durabilidad de caudales menores a $2.27 \mathrm{~m}^{3} / \mathrm{s}$ en porcentajes de tiempo mayores al 50\%. Si la oferta de caudal de agua es mayor que la demanda de agua, entonces la fuente proveedora tendrá la capacidad de satisfacer la demanda sin necesidad de almacenamiento, situación que no se presenta para el caso de la microcuenca Apacheta ya que el caudal es menor en un $50 \%$ de tiempo, por lo que es necesario proponer programas de almacenamiento de agua.

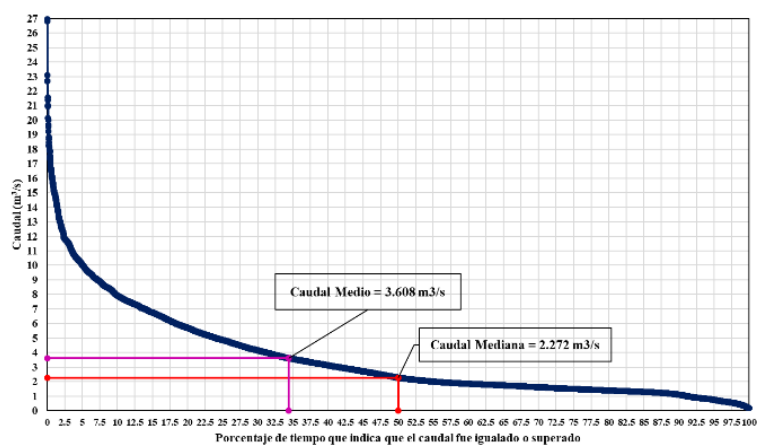

Figura 6. Curva de Duración de Flujo para el caudal de salida simulado con PRMS-IV en la bocatoma Apacheta, del año 2000 al 2018.

El caudal medio mensual simulado con PRMS-IV en la microcuenca corresponde al aporte de las 31 Unidades de Respuesta Hidrológica (HRUs) que la conforman, cuyos tributarios convergen a la zona más baja de la microcuenca, dando lugar al nacimiento del río Apacheta. En la serie de tiempo de la Figura 7 se observa una pronunciada tendencia negativa del caudal de salida desde enero del año 2000 a diciembre de 2018, lo cual implica una significativa disminución del caudal en los últimos años.

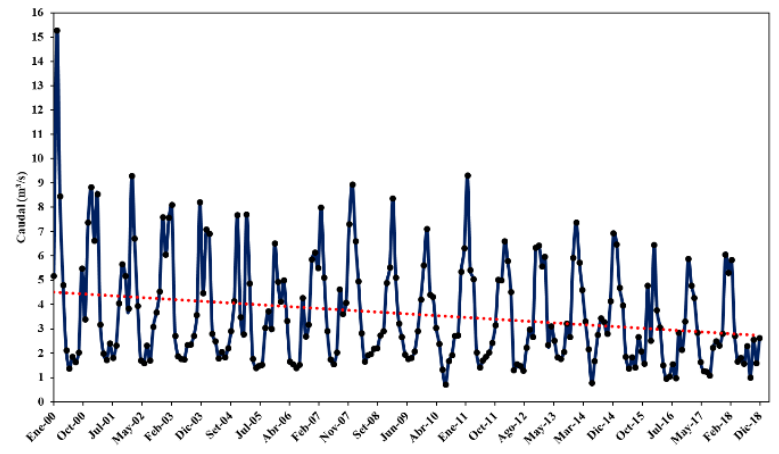

Figura 7. Serie de tiempo del caudal medio mensual de salida simulado con el modelo PRMS-IV en la microcuenca Apacheta, desde el año 2000 al 2018.

En la Figura 8 se muestra el diagrama de cajas del caudal medio mensual de salida en la microcuenca, desde el año 2000 al 2018. En ella se observa la distribución estacional del caudal medio mensual, el mismo que muestra caudales con mayor afluente en los meses de diciembre, enero, febrero y marzo, que corresponden a los meses del estadío lluvioso; los valores atípicos de hasta $15 \mathrm{~m}^{3} / \mathrm{s}$ se deben a precipitaciones intensas fortuitas. $\mathrm{El}$ caudal medio mensual de salida con menor valor se da en los meses de mayo, junio, julio y agosto, con valores atípicos de hasta $2.5 \mathrm{~m}^{3} / \mathrm{s}$ y corresponden a meses de un estadío seco, con precipitaciones de tipo nival (Moncada et al., 2020).

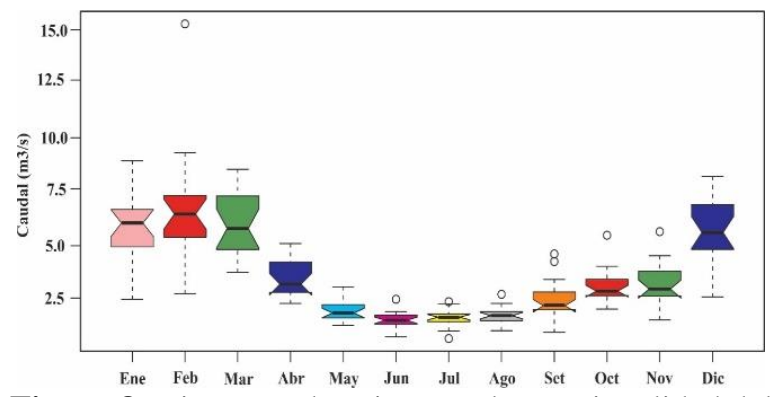

Figura 8. Diagrama de cajas para la estacionalidad del caudal medio mensual de salida en la microcuenca Apacheta.

La Figura 9 muestra la descomposición de la serie de tiempo del caudal medio mensual de salida en la microcuenca en tres componentes: estacional, tendencia y residuo. El componente estacional se asume que es constante para cada año, por lo que se demuestra que la evolución del caudal medio mensual, con el paso de los años, mantiene la existencia de un patrón de evolución. El componente de tendencia muestra un pronunciado decremento en la evolución del caudal medio mensual desde el año 2000 al 2006, pero en el 2007 se observa un ligero incremento que con el paso de los años va disminuyendo, acentuándose en un significativo decremento desde el 2008 hasta el 2015. En los últimos años, entre el 2015 al 2018, el 
caudal de salida es mucho menor que en los años anteriores, por lo que se podría inferir que la serie de tendencia que se presenta desde el año 2000 al 2018 es "decreciente". En el componente de residuo o irregularidad o ruido se muestra la presencia de muchas variaciones, lo que implica la existencia de estacionariedad, justificando el diagrama de cajas de la Figura 8.

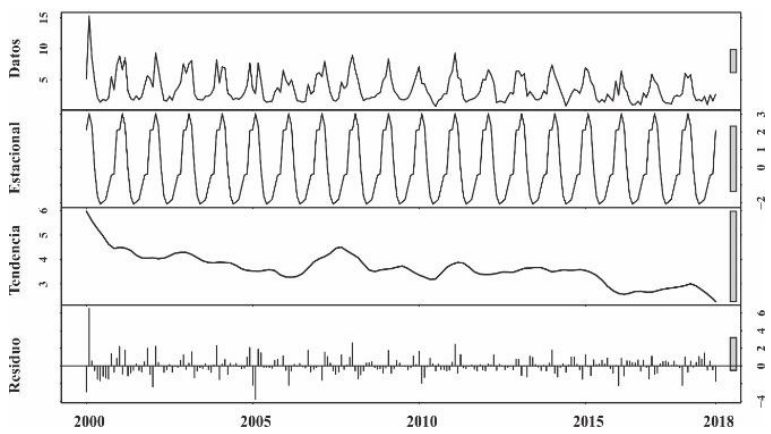

Figura 9. Descomposición de la serie de tiempo del caudal medio mensual de salida simulado con PRMSIV en la microcuenca Apacheta, desde el año 2000 al 2018.

La tendencia anual del caudal medio anual (Q) en la microcuenca, desde el año 2000 al 2018, sigue el comportamiento del modelo determinado por la Fórmula 1, conforme se muestra en la Figura 10. En el año 2000 el valor medio del caudal fue de $4.33 \mathrm{~m}^{3} / \mathrm{s}$ y en el 2018 fue de $2.77 \mathrm{~m}^{3} / \mathrm{s}$. Siguiendo el comportamiento de la línea de tendencia anual, desde el 2000 al 2018, el caudal medio anual de salida disminuyó en $1.56 \mathrm{~m}^{3} / \mathrm{s}$, equivalente al $36.1 \%$ con respecto a su valor inicial, en 19 años, lo cual justifica una variación considerable de algunos parámetros hidrológicos y climáticos, como la disminución de la precipitación, el incremento de la temperatura superficial del suelo, el aumento de la evapotranspiración, la disminución de la cobertura nival o el incremento de la temperatura máxima del aire en la microcuenca Apacheta.

$$
Q=-0.0868 \times(\text { Año })+177.93
$$

Fórmula 1. Tendencia anual del caudal medio anual (Q).

El pronóstico de la serie de tiempo del caudal medio mensual de salida en la microcuenca Apacheta se representa en la Figura 11, donde se observa que los datos ajustados al modelo siguen el comportamiento de la línea de color azul, los datos modelados para el pronóstico siguen la estacionalidad de la línea de color verde, los datos pronosticados desde enero de 2019 hasta diciembre de 2020 siguen el comportamiento de la línea de color rojo y la sombra de color gris corresponde al rango de probabilidad de los datos pronosticados. Así mismo, se observa que el modelo de pronóstico del caudal de salida, de acuerdo con los resultados mostrados en la Figura 11, sigue el mismo comportamiento de la línea de tendencia anual mostrada en la Figura 10. Es importante resaltar que las predicciones se realizan dentro del plazo de 5 años, ya que es el tiempo necesario para la toma de decisiones por parte de las autoridades competentes, priorizando a nivel del Gobierno Regional de Ayacucho una cartera de proyectos relacionados con la siembra y cosecha del agua de acuerdo con el Reglamento de la Ley $N^{\circ} 30215$, Ley de Mecanismos de Retribución por Servicios Ecosistémicos (MINAM, 2016), y la priorización de áreas para el programa Sierra Azul.

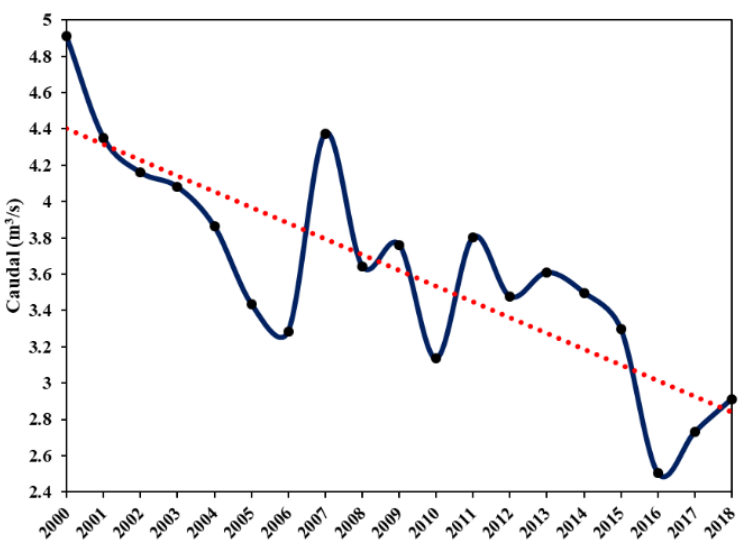

Figura 10. Tendencia anual del caudal medio anual simulado con PRMS-IV en la microcuenca Apacheta, desde el año 2000 al 2018.

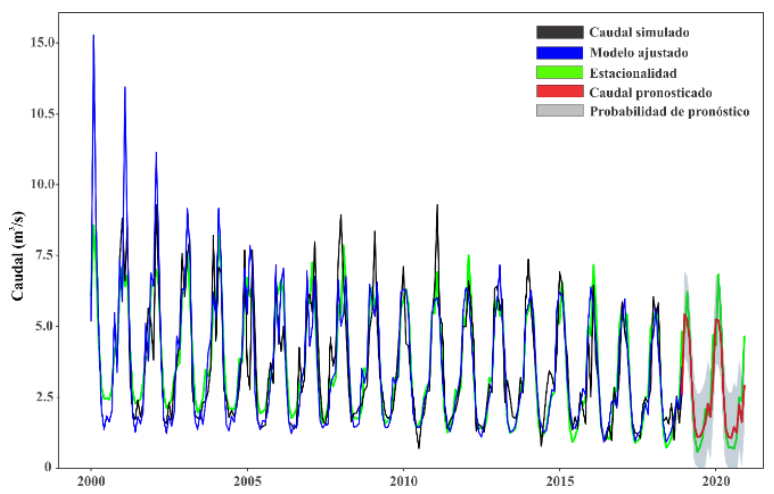

Figura 11. Pronóstico del caudal medio mensual de salida simulado con PRMS-IV en la microcuenca Apacheta, desde enero de 2000 a diciembre de 2018.

La Figura 12 compara la estacionalidad del caudal de salida en referencia al caudal ecológico de la microcuenca Apacheta; ambos mantienen un patrón similar de comportamiento para los doce meses del año. A pesar de ello, el caudal de salida se acerca al caudal ecológico en los meses de mayo, junio, julio y agosto, lo cual indica que en estos meses el caudal de salida es muy bajo, con una tendencia de escasez de agua, ya que estos meses corresponden al estadío seco. La determinación del caudal ecológico garantiza un caudal necesario para la conservación de la biodiversidad y el 
mantenimiento de los ecosistemas de la microcuenca. Considerando que la bocatoma Apacheta fue construida en el año 1985, no tiene las consideraciones de los parámetros impuestos en la normativa de la Resolución Jefatural 267-2019-ANA, (ANA， 2019), lo cual evidencia la no sostenibilidad del trasvase a la presa de Cuchoquesera, siendo preocupante en los últimos años, tal como se demuestra en la evaluación de la tendencia anual y el pronóstico del caudal de salida en la microcuenca Apacheta.

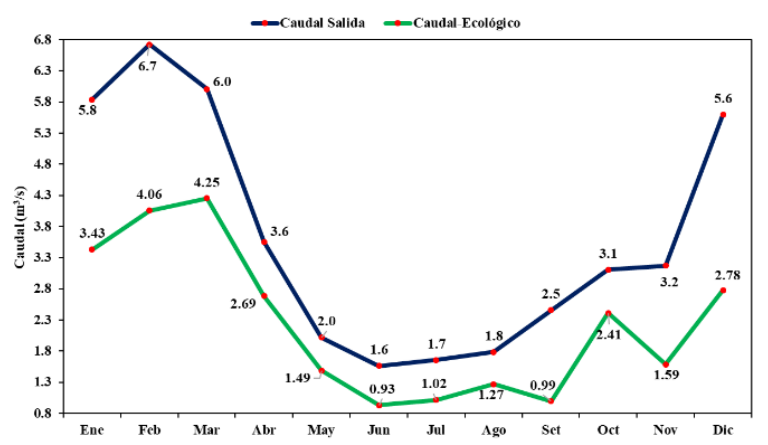

Figura 12. Comparación del caudal de salida estacional con el caudal ecológico de la microcuenca Apacheta.

\section{Conclusiones}

Las pruebas de validación realizadas al caudal de salida simulado con el modelo PRMS-IV con respecto a los aforos medidos en la bocatoma Apacheta, mostraron valores dentro del rango de evaluación muy cerca al valor ideal, correspondiente a cada indicador de validación, por lo que se concluye que los valores del caudal simulado mediante la aplicación del modelo PRMS-IV, son aceptables, con una ligera sobrestimación de los datos del caudal simulado con respecto al observado.

La curva de duración del flujo de caudal de salida en la primera bocatoma Apacheta es un indicativo del proceso de drenaje en la microcuenca, mostrando un caudal medio de $3.61 \mathrm{~m}^{3} / \mathrm{s}$ mayor al caudal mediano $2.27 \mathrm{~m}^{3} / \mathrm{s}$, lo que significa que existen caudales mayores a $3.61 \mathrm{~m}^{3} / \mathrm{s}$ en porcentajes menores a $34.4 \%$. Por ello, se concluye que existe mayor durabilidad para caudales menores a $2.27 \mathrm{~m}^{3} / \mathrm{s}$ en porcentajes mayores al $50 \%$. Asimismo, el caudal mínimo probable de la curva se puede suministrar durante todo el año, con una probabilidad de excedencia próxima al $100 \%$.

La tendencia anual del caudal de salida en la microcuenca Apacheta sigue el comportamiento lineal con tendencia negativa, mostrando un decremento de $1.56 \mathrm{~m}^{3} / \mathrm{s}$ desde el año 2000 al 2018, que representa una disminución del $36.1 \%$ del valor inicial. Si se mantiene la línea de tendencia negativa, se espera que a finales del 2020 el caudal medio anual de salida sea de 2.59 $\mathrm{m}^{3} / \mathrm{s}$, correspondiente a una disminución del $40.1 \%$; a finales del 2023 se espera que el caudal medio anual de salida sea de $2.33 \mathrm{~m}^{3} / \mathrm{s}$, con una disminución del $46.1 \%$ muy cerca al caudal ecológico que es $2.24 \mathrm{~m}^{3} / \mathrm{s}$. En base a estos posibles escenarios y teniendo en cuenta el crecimiento poblacional en la ciudad de Ayacucho, se estima que a finales del año 2025 el caudal medio anual se encontrará por debajo del caudal ecológico. Considerando que la oferta de agua disminuya y que la demanda debido al crecimiento poblacional aumente, se espera una probable escasez de agua en la microcuenca Apacheta, ya que no tendrá la capacidad de satisfacer dicha demanda, por lo que se hace necesario la construcción de presas de almacenamiento de agua o el fortalecimiento de programas de siembra y cosecha de agua, o la búsqueda de fuentes de agua subterránea. Estos resultados, que por ciertos son de escasos trabajos de investigación de este tipo realizados en Ayacucho, son de gran importancia en la medida que los tomadores de decisiones se interesen en hacer frente a los posibles escenarios de escasez de agua que se avizoran dentro del horizonte temporal en un rango de 5 a 10 años.

\section{Agradecimientos}

Este trabajo ha sido posible gracias al apoyo de los proyectos "Strengthening resilience of Andean river basin headwaters facing global change" (PGA_084063), financiado por el Programa PEER de USAID; e "International Water Security Network", financiado por el Lloyd's Register Foundation. Asimismo, los autores agradecen al Dr. Wouter Buytaert por su aporte, al equipo Agua Andes por su apoyo, a OPEMAN del Gobierno Regional de Ayacucho por facilitar los datos hidro meteorológicos, y a la Universidad Nacional de San Cristóbal de Huamanga por la licencia brindada por capacitación.

\section{Literatura citada}

ANA (Autoridad Nacional del Agua). 2019. Resolución Jefatural Nro 267-2019: Aprobación de lineamientos generales para determinar caudales ecológicos. Diario Oficial El Peruano (Normas Legales), XXXVI (1517): 89. Viernes 6 de diciembre. Lima / Perú. https://busquedas.elperuano.pe/download/full/50KiirON KuAAsuYUVHSGla.

Canty M.J. 2014. Image Analysis, Classification and Change Detection in Remote Sensing: With Algorithms for ENVI/IDL and Python, Third Edition. ed. CRC Press, New York.

Chang H. \& Jung I.-W. 2010. Spatial and temporal changes in runoff caused by climate change in a complex large river basin in Oregon. J. Hydrol., 388(3-4): 186-207. DOI: https://doi.org/10.1016/j.jhydrol.2010.04.040.

Cook A.J., Murray T., Luckman A., Vaughan D.G. \& Barrand N.E. 2012. A new 100-m Digital Elevation Model of the Antarctic Peninsula derived from ASTER Global DEM: methods and accuracy assessment. Earth Syst. Sci. Data 4, 129-142. DOI: https://doi.org/10.5194/essd-4-1292012.

Corporación de Fomento y Desarrollo Económico y Social de Ayacucho. 1983. Proyecto Integral del río Cachi: estudio de factibilidad (Proyecto). Ayacucho-Perú. 
Dozier J. 1989. Spectral signature of alpine snow cover from the Landsat thematic mapper. Remote Sens. Environ. 28, 9-22. DOI: https://doi.org/10.1016/00344257(89)90101-6.

Duan Q., Sorooshian S. \& Gupta V.K. 1994. Optimal use of the SCE-UA global optimization method for calibrating watershed models. J. Hydrol., 158(3-4): 265-284. DOI: https://doi.org/10.1016/0022-1694(94)90057-4.

Huaman Y., Moreira-Turcq P., Espinoza R., Llanos R., Apaéstegui J., Turcq B. \& Willems B. 2020. Influencia de los cambios climáticos en la acumulación de carbono en Bofedales Altoandinos durante los últimos 2500 años. Ecol. Apl., 19(1): 35-41. DOI: https://doi.org/10.21704/rea.v19i1.1444.

Hyndman R., Athanasopoulos G., Bergmeir Ch., Caceres G., Chhay L., O'Hara-Wild M., Petropoulos F., Razbash S., Wang E. \& Yasmeen F. 2020. Package "Forecast". Forecasting functions for time series and linear models. https://pkg.robjhyndman.com/forecast/.

Krause P., Boyle D.P. \& Bäse F. 2005. Comparison of different efficiency criteria for hydrological model assessment. Adv Geosci, 5: 89-97. DOI:

https://doi.org/10.5194/adgeo-5-89-2005.

Kult J., Choi W. \& Choi J. 2014. Sensitivity of the Snowmelt Runoff Model to snow covered area and temperature inputs. Appl. Geogr. 55, 30-38. DOI: https://doi.org/10.1016/j.apgeog.2014.08.011.

LaFontaine J.H., Hay L.E., Viger R.J., Markstrom S.L., Regan R.S., Elliott C.M. \& Jones J.W. 2013. Application of the Precipitation-Runoff Modeling System (PRMS) in the Apalachicola-Chattahoochee-Flint River Basin in the southeastern United States (USGS Numbered Series No. 5162), Scientific Investigations Report. U.S. Geological Survey, United States.

Leavesley G.H., Lichty R.W., Troutman B.M. \& Saindon L.G. 1983. Precipitation-runoff modeling system; user's manual (USGS Numbered Series No. 83-4238), WaterResources Investigations Report. U.S. Geological Survey, Water Resources Division, Denver, Colorado.

Lilliefors H.W. 1967. On the Kolmogorov-Smirnov Test for Normality with Mean and Variance Unknown. J. Am. Stat. Assoc., 62(318): 399-402. DOI: https://doi.org/10.1080/01621459.1967.10482916.

Markstrom S., Niswonger R., Regan R.S., Prudic D. \& Barlow P. 2008. GSFLOW-Coupled Groundwater and Surface-Water Flow Model Based on the Integration of the Precipitation-Runoff Modeling System (PRMS) and the Modular Ground-Water Flow Model (MODFLOW2005). U.S. Geological Survey, Virginia.
Markstrom S., Regan R., Hay L., Viger R., Webb R., Payn R. \& LaFontaine J. 2015. PRMS-IV, the precipitation-runoff modeling system, version 4: U.S. Geological Survey, Reston, Virginia. USGS, United States.

MINAM (Ministerio del Ambiente). 2016. Decreto Supremo No 009-2016-MINAM: Aprobación del Reglamento de la Ley $\mathrm{N}^{\circ} 30215$, "Ley de Mecanismos de Retribución por Servicios Ecosistémicos". Diario Oficial El Peruano (Normas Legales), XXXIII (13745): 593739-593745. Lima / Perú.

https://busquedas.elperuano.pe/download/full/CljZHnW9aw WAX9rwPZcaou.

Moncada W., Pereda A. M. \& Lagos M. 2018. Caracterización de la dinámica espacial de humedales en la cabecera de cuenca Cachi-Apacheta, Región Ayacucho, mediante el uso de imágenes de radar Sentinel 1A. Revista Investigación, 26(1): 173-183. http://revistas.unsch.edu.pe/index.php/investigacion/arti cle/view/245.

Moncada W., Willems B. \& Rojas J. 2020. Estimación de estadíos estacionales a partir de parámetros climáticos medidos en la estación meteorológica de la microcuenca Apacheta, Región Ayacucho, 2000 al 2018. Revista de Investigación de Física, 23(2): 17-25. UNMSM. https://fisica.unmsm.edu.pe/rif/previo_files/20202/03moncada.pdf.

Nash J.E. \& Sutcliffe J.V. 1970. River flow forecasting through conceptual models part I - A discussion of principles. J. Hydrol. 10, 282-290. DOI: https://doi.org/10.1016/0022-1694(70)90255-6.

Pereda A., Moncada W. \& Verde L. 2018. Respuesta nival de la cabecera de cuenca Cachi-Apacheta de Ayacucho. Editorial Académica Española, Perú.

Rouse J., Haas R., Deering D., Schell J. \& Harlan J. 1974. Monitoring the vernal advancement and retrogradation (green wave effect) of natural vegetation. NASA, United States.

Sobrino J.A. 2000. Teledetección. Servicio de publicaciones Universidad de Valencia, Valencia-España.

Taylor S. \& Letham B. 2017. Forecasting at scale. PeerJ Prepr., 525. DOI:https://doi.org/10.7287/peerj.preprints.3190v2.

Teng F., Huang W., Cai Y., Zheng C. \& Zou S. 2017. Application of Hydrological Model PRMS to Simulate Daily Rainfall Runoff in Zamask-Yingluoxia Subbasin of the Heihe River Basin. Water, 9(10): 769:1-14. DOI: https://doi.org/10.3390/w9100769.

Tornero J. 2017. Introducción al Forecasting con R Statistics. Dr. https://www.doctormetrics.com/introduccion-alforecasting-con-r-statistics/. 
Tabla 1. Cálculo del caudal ecológico para la microcuenca Apacheta, Región Ayacucho, Perú.

\begin{tabular}{rlllllllllllll}
\hline $\mathbf{m}$ & $\mathbf{P}(\boldsymbol{\%})$ & Ene & Feb & Mar & Abr & May & Jun & Jul & Ago & Set & Oct & Nov & Dic \\
\hline 1 & 5.26 & 8.94 & 15.27 & 8.54 & 5.10 & 3.10 & 2.52 & 2.41 & 2.75 & 4.62 & 5.48 & 5.65 & 8.21 \\
2 & 10.53 & 8.82 & 9.30 & 8.44 & 5.04 & 2.91 & 1.94 & 2.32 & 2.32 & 4.27 & 4.05 & 4.53 & 7.68 \\
3 & 15.79 & 7.37 & 9.29 & 8.09 & 4.87 & 2.67 & 1.92 & 2.05 & 2.29 & 3.43 & 3.72 & 4.21 & 7.58 \\
4 & 21.05 & 7.11 & 8.36 & 7.99 & 4.79 & 2.48 & 1.80 & 1.95 & 2.17 & 3.09 & 3.67 & 4.15 & 7.37 \\
5 & 26.32 & 6.92 & 7.56 & 7.70 & 4.52 & 2.38 & 1.79 & 1.85 & 2.02 & 3.03 & 3.60 & 4.06 & 7.29 \\
6 & 31.58 & 6.42 & 7.08 & 6.91 & 3.96 & 2.15 & 1.77 & 1.82 & 1.86 & 2.67 & 3.27 & 3.57 & 6.51 \\
7 & 36.84 & 6.32 & 6.61 & 6.72 & 3.94 & 2.12 & 1.74 & 1.82 & 1.83 & 2.36 & 3.21 & 3.39 & 6.33 \\
8 & 42.11 & 6.13 & 6.61 & 5.97 & 3.32 & 2.03 & 1.72 & 1.77 & 1.81 & 2.30 & 2.97 & 3.17 & 5.91 \\
9 & 47.37 & 6.05 & 6.61 & 5.83 & 3.29 & 1.97 & 1.58 & 1.74 & 1.81 & 2.23 & 2.90 & 3.14 & 5.86 \\
10 & 52.63 & 6.05 & 6.46 & 5.80 & 3.22 & 1.88 & 1.54 & 1.69 & 1.76 & 2.22 & 2.90 & 2.99 & 5.61 \\
11 & 57.89 & 5.87 & 6.45 & 5.40 & 3.17 & 1.85 & 1.54 & 1.68 & 1.70 & 2.21 & 2.85 & 2.90 & 5.34 \\
12 & 63.16 & 5.51 & 5.71 & 5.10 & 3.05 & 1.76 & 1.40 & 1.55 & 1.67 & 2.20 & 2.74 & 2.80 & 5.17 \\
13 & 68.42 & 5.18 & 5.56 & 5.00 & 3.03 & 1.69 & 1.39 & 1.55 & 1.64 & 2.06 & 2.72 & 2.73 & 5.01 \\
14 & 73.68 & 4.99 & 5.50 & 4.94 & 2.84 & 1.66 & 1.37 & 1.48 & 1.53 & 2.05 & 2.70 & 2.67 & 4.88 \\
15 & 78.95 & 4.92 & 5.30 & 4.70 & 2.83 & 1.65 & 1.36 & 1.48 & 1.52 & 2.03 & 2.68 & 2.67 & 4.78 \\
16 & 84.21 & 4.48 & 4.78 & 4.60 & 2.79 & 1.64 & 1.32 & 1.39 & 1.51 & 2.02 & 2.55 & 2.32 & 4.13 \\
17 & 89.47 & 3.82 & 4.40 & 4.31 & 2.72 & 1.62 & 1.25 & 1.24 & 1.41 & 1.93 & 2.48 & 2.13 & 3.31 \\
18 & 94.74 & 3.48 & 4.12 & 4.28 & 2.71 & 1.50 & 0.94 & 1.04 & 1.28 & 1.00 & 2.43 & 1.59 & 2.79 \\
19 & 100.00 & 2.51 & 2.78 & 3.76 & 2.32 & 1.31 & 0.78 & 0.70 & 1.07 & 0.98 & 2.06 & 1.56 & 2.63 \\
\hline \multicolumn{2}{r}{ Promedio } & 5.84 & 6.72 & 6.00 & 3.55 & 2.02 & 1.56 & 1.66 & 1.79 & 2.46 & 3.10 & 3.17 & 5.60
\end{tabular}

Tabla 2. Caudal ecológico al 95\% de la microcuenca Apacheta, Región Ayacucho, Perú.

\begin{tabular}{cccccccccccccr}
\hline P (\%) & Ene & Feb & Mar & Abr & May & Jun & Jul & Ago & Set & Oct & Nov & Dic & Prom $\left(\mathbf{m}^{3} / \mathbf{s}\right)$ \\
\hline 94.74 & 3.48 & 4.12 & 4.28 & 2.71 & 1.50 & 0.94 & 1.04 & 1.28 & 1.00 & 2.43 & 1.59 & 2.79 & -- \\
95 & 3.43 & 4.06 & 4.25 & 2.69 & 1.49 & 0.93 & 1.02 & 1.27 & 0.99 & 2.41 & 1.59 & 2.78 & $\mathbf{2 . 2 4}$ \\
100 & 2.51 & 2.78 & 3.76 & 2.32 & 1.31 & 0.78 & 0.70 & 1.07 & 0.98 & 2.06 & 1.56 & 2.63 & -- \\
\hline
\end{tabular}

Tabla 3. Pruebas de validación del caudal simulado con el modelo PRMS-IV con respecto al caudal observado en el punto de aforo en la Bocatoma Apacheta.

\begin{tabular}{llll}
\hline Indicador & Rango de Evaluación & Valor Ideal & Valor Real \\
\hline Eficiencia de Nash-Sutcliffe & {$[-\infty ; 1]$} & 1 & 0.89 \\
Coeficiente de Nash-Sutcliffe-Ln & {$[-\infty ; 1]$} & 1 & 0.93 \\
Coeficiente de correlación Pearson & {$[-1 ; 1]$} & 1 & 0.96 \\
Coeficiente de determinación R ${ }^{2}$ & {$[0 ; 1]$} & 1 & 0.91 \\
Raíz del error medio cuadrático RMSE & {$[0 ;+\infty]$} & 0 & 0.25 \\
Bias-Score (BS) & {$[0 ; 1]$} & 1 & 0.99 \\
PBias & {$[-\infty ;+\infty]$} & 0 & 0.12 \\
Error Relativo al volumen (RVE) & {$[-\infty ;+\infty]$} & 0 & 0.07 \\
Error de valores picos normalizados (NPE) & {$[-\infty ;+\infty]$} & 0 & 0.19 \\
\hline
\end{tabular}


Apéndice 1. Código en Matlab para el cálculo del volumen debajo de la curva de duración del caudal de salida en la microcuenca Apacheta.

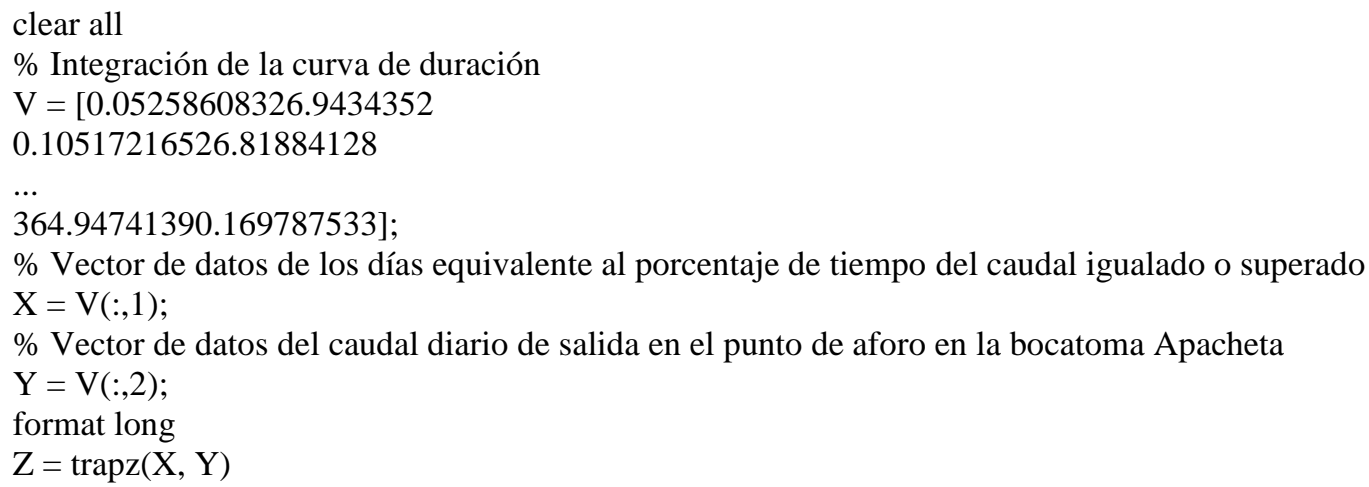

Apéndice 2. Código para el pronóstico del caudal medio mensual de salida en la microcuenca Apache setwd("D:/dirección de ubicación de la base de datos")

library("TTR")

require(graphics)

a <- read.csv("Caudal_Mensual_Apa.csv", header=T, dec=".", sep=";")

$\mathrm{Q}<-$ as.numeric(a $\$$ Caudal)

Qst <- ts(Q, start $=\mathrm{c}(2000,1)$, end $=\mathrm{c}(2018,12)$, freq $=12)$

library(forecast)

df_arima $<-$ ts $($ Qst, frequency $=12$, start $=\mathrm{c}(2000,1)$, end $=\mathrm{c}(2018,12))$

model <- forecast::auto.arima(df_arima)

m.forecast <- forecast::forecast(model, $h=24$ )

library(prophet)

df_prophet=data.frame(seq(as.Date("2000-01-01"), as.Date("2018-12-31"), "months"), df_arima)

names(df_prophet) = c("ds", "y")

$\mathrm{m}=$ prophet(df_prophet)

future_df_prophet $=$ make_future_dataframe $(\mathrm{m}$, periods $=24$, freq $=$ 'months' $)$

prophet.forecast $=$ predict $(\mathrm{m}$, future_df_prophet $)$

library(ggplot2)

library(ggfortify)

fortify.forecast <- fortify (m.forecast, ts.connect = TRUE)

names(fortify.forecast) <- c('ds', 'data', 'fitted', 'yhat', 'Lo.80', 'Hi.80', 'Lo.90', 'Hi.90')

library(ggplot2)

$\operatorname{ggplot}($ data $=$ prophet.forecast, aes $(\mathrm{x}=$ as.Date $(\mathrm{ds}), \mathrm{y}=\mathrm{yhat}))+$ \#prophet

geom_line $(\mathrm{col}=$ 'green') +

geom_line $($ data $=$ fortify.forecast, aes $(\mathrm{x}=\mathrm{ds}, \mathrm{y}=$ data $)$, col='black' $)+$

geom_line $($ data $=$ fortify.forecast, aes $(\mathrm{x}=\mathrm{ds}, \mathrm{y}=$ fitted $)$, color='blue' $)+$

geom_line $($ data $=$ fortify.forecast, aes $(\mathrm{x}=\mathrm{ds}, \mathrm{y}=\mathrm{yhat})$, color='red' $)+$

geom_ribbon $($ data $=$ fortify.forecast, $\operatorname{aes}(\mathrm{x}=\mathrm{ds}, \mathrm{ymin}=$ Lo.80, ymax $=$ Hi.80), fill $=$ 'skyblue4', alpha=0.3)

1 Universidad Nacional San Cristóbal de Huamanga / Ayacucho / Perú. Autor de correspondencia: wilmer.moncada@unsch.edu.pe.

${ }^{2}$ Centro de Competencias del Agua / Lima / Perú. bwillems@cca.org.pe. 